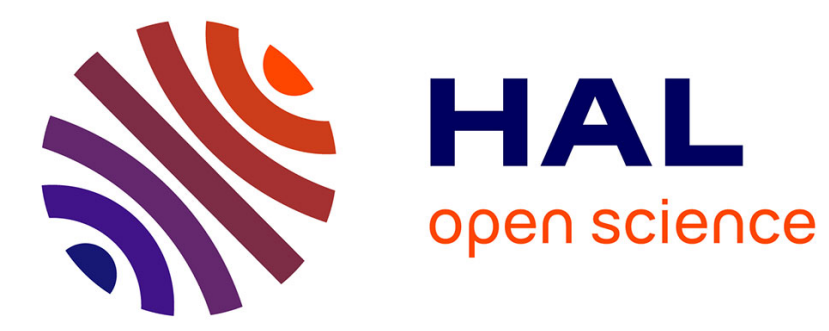

\title{
Optically Induced Phase Change for Magnetoresistance Modulation
}

Guodong Wei, Xiaoyang Lin, Zhizhong Si, Dong Wang, Xinhe Wang, Xiaofei Fan, Kun Deng, Kaï Liu, Kaili Jiang, Na Lei, et al.

\section{- To cite this version:}

Guodong Wei, Xiaoyang Lin, Zhizhong Si, Dong Wang, Xinhe Wang, et al.. Optically Induced Phase Change for Magnetoresistance Modulation. Advanced Quantum Technologies, 2020, 10.1002/qute.201900104 . hal-02860979

\section{HAL Id: hal-02860979 \\ https://hal.univ-lorraine.fr/hal-02860979}

Submitted on 10 Feb 2021

HAL is a multi-disciplinary open access archive for the deposit and dissemination of scientific research documents, whether they are published or not. The documents may come from teaching and research institutions in France or abroad, or from public or private research centers.
L'archive ouverte pluridisciplinaire HAL, est destinée au dépôt et à la diffusion de documents scientifiques de niveau recherche, publiés ou non, émanant des établissements d'enseignement et de recherche français ou étrangers, des laboratoires publics ou privés.

\section{(c)(1)}

Distributed under a Creative Commons Attribution| 4.0 International License 


\title{
Optically Induced Phase Change for Magnetoresistance Modulation
}

\author{
Guodong Wei, Xiaoyang Lin,* Zhizhong Si, Dong Wang, Xinhe Wang, Xiaofei Fan, \\ Kun Deng, Kai Liu, Kaili Jiang, Na Lei, Yanxue Chen, Stephane Mangin, Eric Fullerton, \\ and Weisheng Zhao*
}

Optical methods for magnetism manipulation have been considered as a promising strategy for ultralow-power and ultrahigh-speed data storage and processing, which have become an emerging field of spintronics. However, a widely applicable and efficient method has rarely been demonstrated. Here, the strongly correlated electron material vanadium dioxide $\left(\mathrm{VO}_{2}\right)$ is used to realize the optically induced phase change for control of the magnetism in NiFe. The $\mathrm{NiFe} / \mathrm{VO}_{2}$ bilayer heterostructure features appreciable modulations of electrical conductivity $(32 \%)$, coercivity $(37.5 \%)$, and magnetic anisotropy $(25 \%)$. Further analyses indicate that interfacial strain coupling plays a crucial role in the magnetic modulation. Utilizing this heterostructure, which can respond to both optical and magnetic stimuli, a phase change controlled anisotropic magnetoresistance (AMR) device is fabricated, and reconfigurable Boolean logics are implemented. As a demonstration of phase change spintronics, this work may pave the way for next-generation opto-electronics in the post-Moore era. modulations of magnetism, especially those achieved by local control methods such as voltage-controlled magnetic anisotropy, ${ }^{[4,5]}$ all-optical switching, ${ }^{[6,7]}$ multiferroic, ${ }^{[8,9]}$ and ionic ${ }^{[10-12]}$ modulations, have already demonstrated great potential in device performance improvements and application revolutions..$^{[13]}$ Meanwhile, optical devices with the advances of high speed, wide bandwidth, and low loss have greatly promoted the modern communication industry. ${ }^{[14]} \mathrm{Com}$ bining the advantages of spintronic and opto-electronic devices may highly improve the energy conversion efficiency, increase the device speed, and diversify the functionalities. ${ }^{[15,16]}$ In this sense, a strategy to realize the optical control of magnetic characteristics can promote the integration of spintronic devices into optical systems and trigger emergent device applications.

Strong correlations between electrons enable extraordinary control of physical properties (e.g., magnetism and conductivity) via different strategies (e.g., electric fields, magnetic fields, and stresses), which implies numerous possibilities for strongly correlated electron materials in various research fields, including high-temperature superconductors, 2D electron gas systems,
Dr. G. Wei, Prof. X. Lin, Z. Si, Dr. X. Wang, X. Fan, K. Deng, Prof. N. Lei, Prof. W. Zhao

Fert Beijing Research Institute, School of Microelectronics \& Beijing Advanced Innovation Center for Big Data and Brain Computing (BDBC)

Beihang University

Beijing 100191, China

E-mail:XYLin@buaa.edu.cn; weisheng.zhao@buaa.edu.cn

Prof. X. Lin, Prof. W. Zhao

Beihang-Goertek Joint Microelectronics Institute, Qingdao Research Institute

Beihang University

Qingdao 266000, China

Dr. D. Wang, Prof. Y. Chen

School of Physics, State Key Laboratory of Crystal Materials

Shandong University

Jinan 250100, China

The ORCID identification number(s) for the author(s) of this article can be found under https://doi.org/10.1002/qute.201900104

DOI: 10.1002/qute.201900104
Prof. K. Jiang

State Key Laboratory of Low-Dimensional Quantum Physics

Department of Physics \& Tsinghua-Foxconn Nanotechnology Research Center

Collaborative Innovation Center of Quantum Matter

Tsinghua University

Beijing 100084, China

Prof. K. Liu

State Key Laboratory of New Ceramics and Fine Processing, School of

Materials Science \& Engineering

Tsinghua University

Beijing 100084, China

Prof. S. Mangin

Institut Jean Lamour, UMR 7198

CNRS-Université de Lorraine

F-54000 Nancy, France

Prof. E. Fullerton

Center for Memory and Recording Research

University of California San Diego

9500 Gilman Drive, La Jolla, CA 92093, USA 
(a)

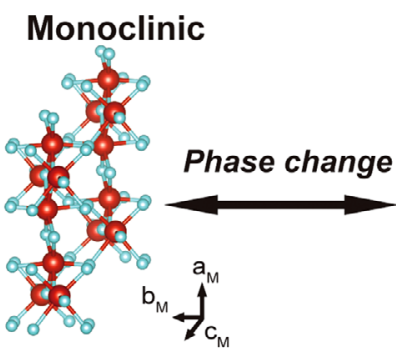

(b)

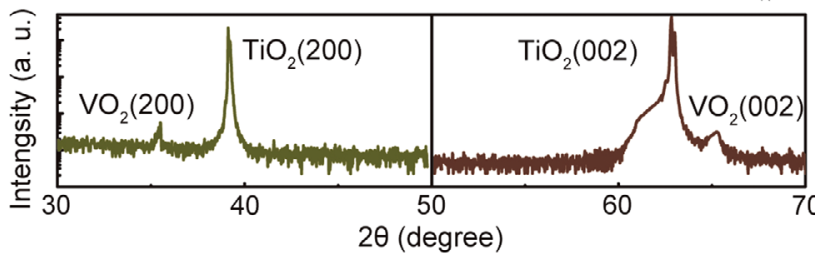

Rutile

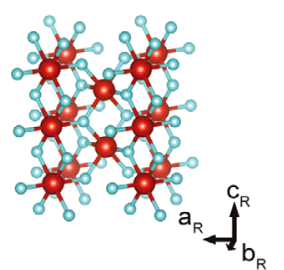

Figure 1. Phase change of $\mathrm{VO}_{2}$ and characterization of the $\mathrm{NiFe} / \mathrm{VO}_{2}$ heterostructure. a) Schematic illustration of the reversible phase change of $\mathrm{VO}$, between monoclinic (M1) and rutile (R) lattice structures. b) XRD $\theta-2 \theta$ scan of the $\mathrm{NiFe} / \mathrm{VO}_{2}$ heterostructure grown on $\mathrm{TiO}_{2}(100)$ and $\mathrm{TiO}_{2}(001)$ substrates, suggesting epitaxial growth of the $\mathrm{VO}_{2}$ layer and polycrystalline growth of the NiFe layer. c) $\mathrm{M}-\mathrm{H}$ hysteresis loops of the $\mathrm{NiFe}(5 \mathrm{~nm}) / \mathrm{VO}_{2}$ $(20 \mathrm{~nm}) / \mathrm{TiO}_{2}(100)$ sample measured before and after the phase change of $\mathrm{VO}_{2}$ (MS at $300 \mathrm{~K}$ and $\mathrm{RS}$ at $360 \mathrm{~K}$ ). The magnetic field is applied perpendicular (data plotted as circles) and parallel (data plotted as squares) to the surface. The insets give the scanning results over small fields.

and next-generation spintronics. ${ }^{[17]}$ As a representative strongly correlated electron material, $\mathrm{VO}_{2}$ exhibits complex property changes as it transforms from a low-temperature monoclinic (M1) insulator into a high-temperature rutile (R) metal at a critical temperature around $340 \mathrm{~K}$ (Figure 1a). ${ }^{[18,19]}$ The phase change can also be induced by other methods such as the electrical gating, ${ }^{[20]}$ optothermal heating (Figure S1, Supporting Information) or even photonic effect, ${ }^{[21]}$ which has greatly enriched its device applications. The sub-ps optically induced phase change further endows $\mathrm{VO}_{2}$-related devices with the feature of ultrafast speed. ${ }^{[22,23]}$ Therefore, a phase change-based spintronic device may reform the magnetism modulation strategy and enable emergent optical-spintronic device applications.

In this work, we study the optical control of magnetism in $\mathrm{NiFe} / \mathrm{VO}_{2}$ heterostructures combining a strongly correlated electron material with a magnetic material. Appreciable and reversible modulations of the electrical conductivity (32\%), coercivity $(37.5 \%)$ and magnetic anisotropy $(25 \%)$ have been achieved in these heterostructures during the phase change of $\mathrm{VO}_{2}$. Further analyses indicate that interfacial strain coupling plays a crucial role in this magnetic property modulation. Based on these features, the heterostructures are further used to demonstrate multiresistance states and implement devices with reconfigurable logics. As an optical magnetism modulation strategy, the collision and blending of strongly correlated electron materials and spintronic materials may pave the way for next-generation optoelectronics.

\section{Magnetism Modulation Induced by $\mathrm{VO}_{2}$ Phase Change}

The $\mathrm{NiFe} / \mathrm{VO}_{2}$ bilayer heterostructure used in this work is prepared by two steps. $\mathrm{VO}_{2}$ is first epitaxially deposited on $\mathrm{TiO}_{2}$ substrates by pulsed laser deposition (PLD), and NiFe is deposited at room temperature by magnetron sputtering. Figure $1 \mathrm{~b}$ shows the $\mathrm{X}$-ray diffraction (XRD) results of the heterostructure samples.

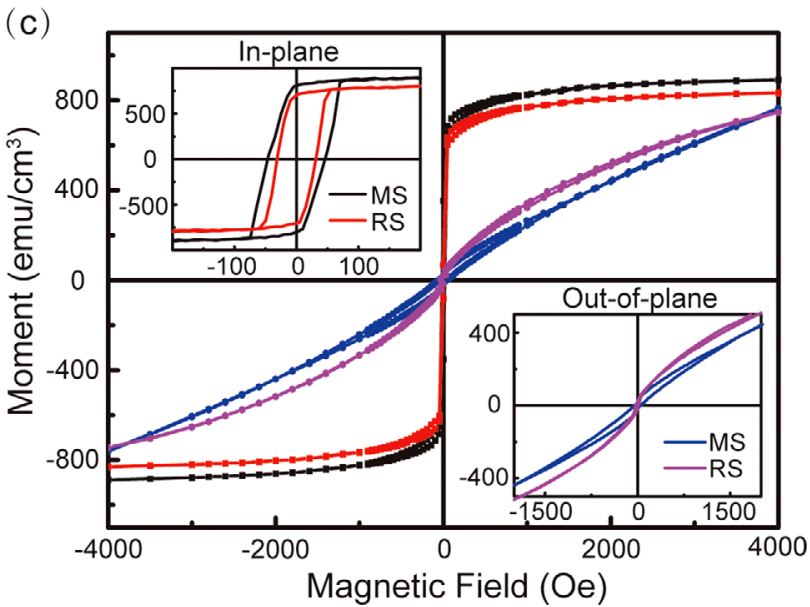

The (200) and (002) peaks of $\mathrm{VO}_{2}$ are detected on the samples with $\mathrm{TiO}_{2}$ (100) and (001) substrates, respectively. The spectrum shows no distinct characteristic peaks of NiFe, which indicates the amorphous feature of NiFe.

To investigate the magnetic characteristics modulated by the phase change, hysteresis loops of a NiFe $(5 \mathrm{~nm}) / \mathrm{VO}_{2}(20 \mathrm{~nm})$ sample grown on a $\mathrm{TiO}_{2}(100)$ substrate are measured for both the monoclinic state (MS, $300 \mathrm{~K}$ ) and rutile state (RS, $360 \mathrm{~K}$ ) of $\mathrm{VO}_{2}$ using a Superconducting Quantum Interference Device (SQUID) magnetometer. As illustrated in Figure 1c, obvious anisotropy can be observed between the results with an in-plane and out-of-plane magnetic field applied. After the $\mathrm{VO}_{2}$ phase change, the saturation magnetization $\left(M_{S}\right)$ of the heterostructure decreases from 898 to $840 \mathrm{emu} \mathrm{cm}^{-3}$, (i.e., a change of $6.5 \%$, calculated as $M_{S}$ difference divided by its original value). The insets show scanning results obtained within a small region, from which the shrinking of the coercive and saturation fields can be detected. Remarkably, the decrease is more obvious out of plane, enhancing the squareness of the curve around the zero field. The coercive field $\left(H_{C}\right)$ decreases from 72 to 45 Oe, (i.e., shows a change of $37.5 \%$, calculated as $H_{C}$ difference divided by its original value). Generally, a temperature increase will not result in such a large change imbalance between $H_{C}$ and $M_{S}$. Further discussion, which will be given in the third section, proves that this variation is different from a mere thermal effect. After excluding the magnetic contribution of $\mathrm{VO}_{2}$ single layer ${ }^{[24]}$ (Figure S1c, Supporting Information), it is reasonable to believe that the effect is mainly caused by the phase change of $\mathrm{VO}_{2}$-induced modulation on NiFe. It is noteworthy that the phase change temperature of $\mathrm{VO}_{2}$ can be tuned from 300 to nearly $400 \mathrm{~K}$ by methods like doping or substrate stress, which will benefit its device application. ${ }^{[25]}$

To further explore this modulation effect, the magnetic parameters of the heterostructure before and after the phase change are determined and summarized in Table 1. The uniaxial magnetic anisotropy $\left(K_{u}\right)$ can be derived from the in-plane and outof-plane hysteresis results, ${ }^{[26]}$ which increases from $-3.55 \times 10^{6}$ to $-2.66 \times 10^{6} \mathrm{erg}^{-3}$. To separate the bulk and interfacial 
Table 1. Magnetic properties of $\mathrm{SiO}_{2}(20 \mathrm{~nm}) / \mathrm{NiFe}(5 \mathrm{~nm}) / \mathrm{VO}_{2}(20 \mathrm{~nm}) /$ $\mathrm{TiO}_{2}(100)$

\begin{tabular}{cccccc} 
Temperature $[\mathrm{K}]$ & $H_{C}[\mathrm{Oe}]$ & $M_{S}\left[\mathrm{emu} \mathrm{cm}^{-3}\right]$ & $H_{K}{ }^{\text {a) }}[\mathrm{Oe}]$ & $K_{u}\left[\mathrm{erg} \mathrm{cm}^{-3}\right]$ & $K_{i}\left[\mathrm{erg} \mathrm{cm}^{-2}\right]$ \\
\hline 300 & 72 & 898 & 4365 & $-3.55 \times 10^{6}$ & 0.755 \\
360 & 45 & 840 & 4229 & $-2.66 \times 10^{6}$ & 0.885
\end{tabular}

a) $H_{K}$ was obtained by extracting the field corresponding to $90 \%$ of the out-of-plane

contributions, the relationship between $K_{u}$ and the NiFe thickness $(t)$ can be written as $K_{u}=K_{b}-2 \pi M_{S}{ }^{2}+K_{i} / t{ }^{[26]}$ where $K_{b}$ is the bulk crystalline anisotropy, $K_{i}$ is the interfacial anisotropy, and $-2 \pi M_{S}{ }^{2}$ is the demagnetizing field. In this convention, $K_{u}<0$ stands for an in-plane easy axis, and its increasing tendency after the phase change indicates an enhancement of the perpendicular magnetic anisotropy. ${ }^{[27]}$ The bulk crystalline anisotropy is negligible because of the amorphous characteristic of the NiFe film. Then, the interfacial magnetic anisotropy can be calculated as $K_{\mathrm{i}}=\left(K_{\mathrm{u}}+2 \pi M_{\mathrm{S}}{ }^{2}\right) t$, which increases from 0.755 to $0.885 \mathrm{erg} \mathrm{cm}^{-2}$. These results indicate the possibility of realizing an interfacial perpendicular magnetic anisotropy enhancement in other similar heterostructures, which is highly desirable for high-density and nonvolatile magnetic storage systems. ${ }^{[26,28]}$

After showing the magnetism modulation effect achieved through the phase change of $\mathrm{VO}_{2}$, we then focus on realizing an optically controlled magnetic modulation by the optothermal effect. To achieve this goal, a laser beam (wavelength $660 \mathrm{~nm}$ ) is used to trigger the phase change. Figure 2a gives the hysteresis loops of the sample under different illumination power measured by an in situ longitudinal magneto-optic Kerr effect (MOKE) magnetometer. An obvious modulation effect can be detected in the power change process, which verifies the optically controlled magnetic modulation feature. Such a modulation effect can be repeated with the same illumination power, reflecting that this process is fully reversible.

To further study the modulation phenomenon, a phase change anisotropic magnetoresistance device (PCAMR device) is fabricated. A schematic illustration of the experimental setup is shown in Figure $2 \mathrm{~b}$. The sample is patterned into a Hall bar to study the magnetic and transport characteristics via magnetoresistance measurements. Figure $2 c$ presents the dependence of the device resistance on the light illumination power. Interestingly, the resistance curves show obvious dependence on applied currents. With small currents ( 1 and $10 \mu \mathrm{A}$ ) applied, the resistance of the heterostructure increases with small illumination power (lower than $0.5 \mathrm{~W} \mathrm{~cm}^{-2}$ ), and starts to decrease when the power becomes larger (higher than $0.5 \mathrm{~W} \mathrm{~cm}^{-2}$ ). For a large measuring current $(100 \mu \mathrm{A})$, the resistance shows a "roller coaster" behavior (inset of Figure 2c). The change rates of the resistance decrease from $32 \%$ to almost $0 \%$ (calculated as resistance difference divided by its original value) as the measuring current is increased. Different from the behavior of ordinary metals, this complex resistance variation can be explained as the competition between the temperature dependent resistance of the magnetic metal and the insulator-to-metal transition induced by the $\mathrm{VO}_{2}$ phase change. Normally, illumination will increase the resistance of the NiFe; while the phase change from insulator to metal will reduce the resistance of $\mathrm{VO}_{2}$. Within the temperature region determined by light illumination and electrical Joule heating, the different resistance-temperature characteristics can thus result in the aforementioned behaviors. As shown in Figure 2c, a small current of $1 \mu \mathrm{A}$ will then be adopted to study the magnetoresistance controlled by the phase change.

Figure $2 \mathrm{~d}-2 \mathrm{f}$ gives the magnetoresistance curves of the device under different illumination power. One obvious variation in this (a)

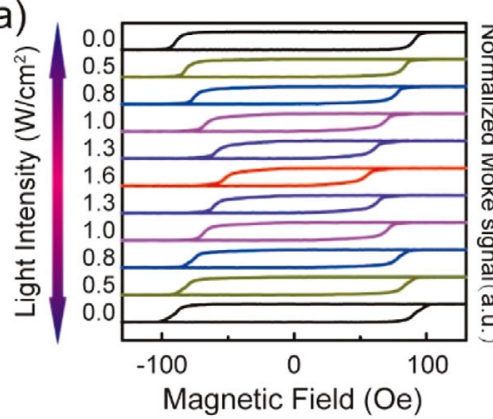

(d)

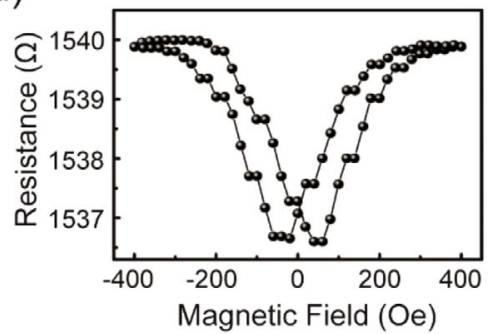

(b)

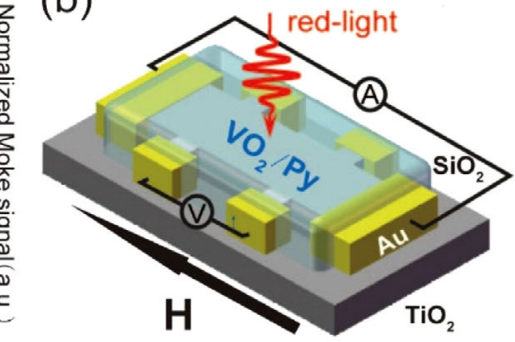

(e)

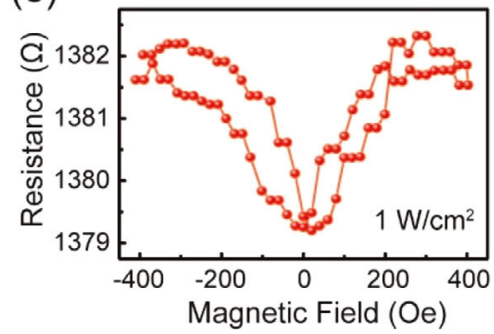

(c)

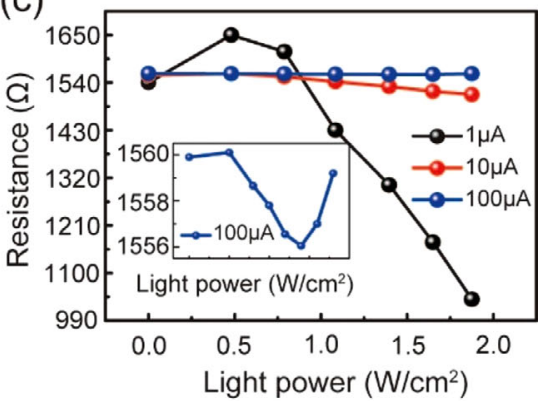

(f)

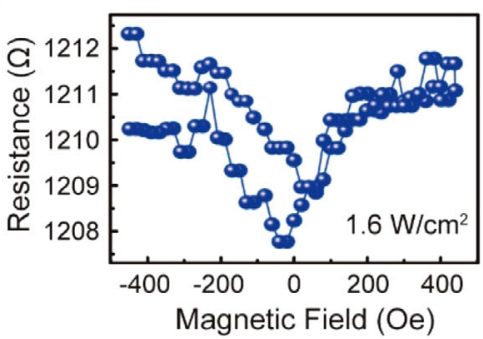

Figure 2. Optically controlled magnetoresistance measurements. a) The hysteresis loops of the sample with different illumination power. b) Schematic drawing of the PCAMR device. Direction of the applied magnetic fields, $H$, are also presented. c) The device resistance change measured with varied applied current under increasing illumination power. d-f) Comparison of the magnetoresistance of the $\mathrm{NiFe}_{(5 \mathrm{~nm}) / \mathrm{VO}}(20 \mathrm{~nm}) / \mathrm{TiO}{ }_{2}(100)$ device without (d) and with the illumination of $1 \mathrm{~W} / \mathrm{cm}^{2}(\mathrm{e})$ and $1.6 \mathrm{~W} / \mathrm{cm}^{2}(\mathrm{f})$ red light. 
(a)

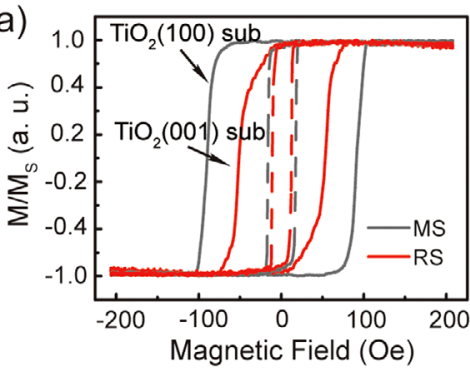

(b)

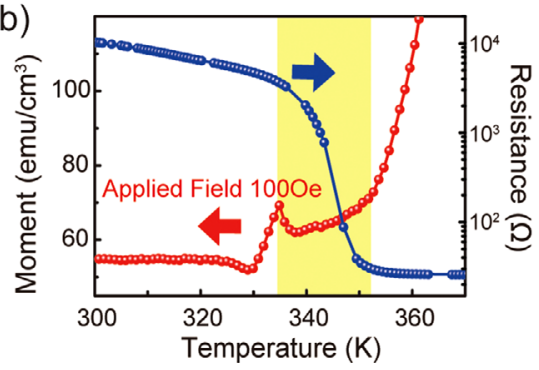

(c)

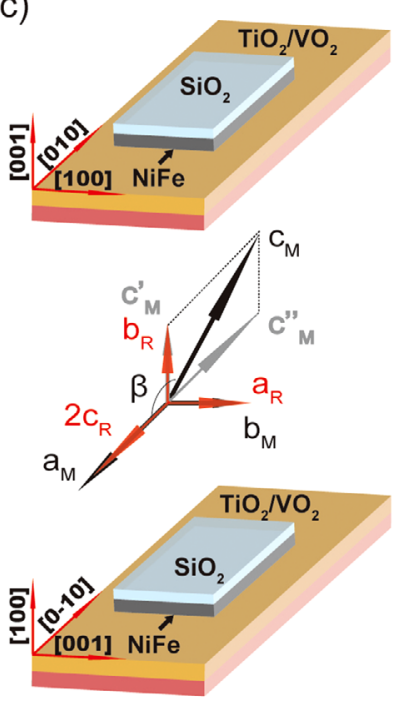

(d)

Monoclinic

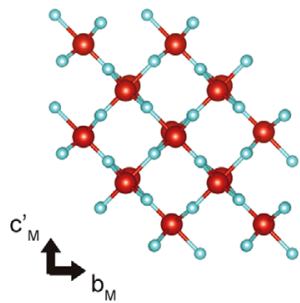

(e)

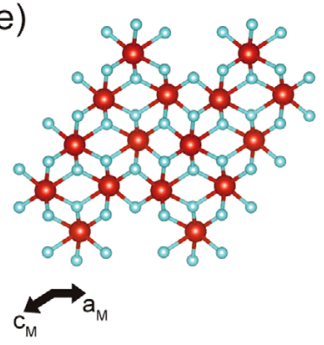

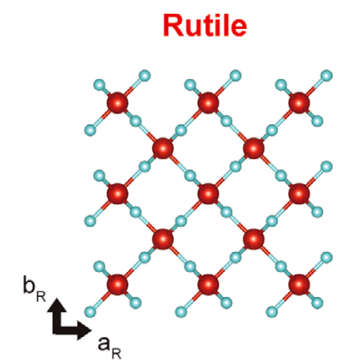

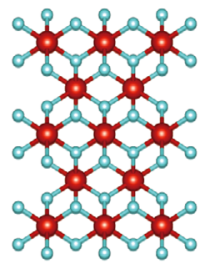<smiles>[13CH3]</smiles>

Figure 3. Interfacial strain analysis and characterization of magnetism modulation. a) Kerr signals of the $\mathrm{NiFe}_{\mathrm{VO}} \mathrm{O}_{2}$ heterostructure grown on different substrates with magnetic field applied in-plane. The $\mathrm{H}_{\mathrm{C}}$ change between the MS and RS is $45 \%$ for the $\mathrm{TiO}_{2}(100)$ substrate and $25 \%$ for the $\mathrm{TiO}_{2}(001)$ substrate when the illumination power reaches $2 \mathrm{~W} \mathrm{~cm}$. b) Temperature dependence of out-of-plane magnetization of the heterostructure with a magnetic field of $100 \mathrm{Oe}$ (the red line), and the resistance change of $\mathrm{VO}_{2}$ from 300 to $360 \mathrm{~K}$ (the blue line). c) Strain analysis when the $\mathrm{VO}_{2}$ phase change occurs. $d$ and e) Schematic diagram of the $\mathrm{VO}_{2}$ crystal lattice change of the (001) plane and (100) plane with regard to the rutile structure.

process is the shift in the "valley bottom" (i.e., from $\approx 50$ to 10 Oe at $1 \mathrm{~W} \mathrm{~cm}$ ), which agrees with the modulation on the coercivity of the heterostructure (Figure 1 and Table 1). In this device, light and magnetic field can induce the insulator-to-metal transition and AMR effects, which endows the device with optically controlled PCAMR feature. Such an effect may enable emergent device applications, which will be discussed in the last section of this paper.

\section{Strain Analysis and Mechanism Explanation}

To clarify the relationship between the magnetism modulation and the phase change of $\mathrm{VO}_{2}$, a series of further experiments are carried out based on the heterostructure samples. As illustrated in Figure 3a, even though the change rates are different, an obvious coercive field decrease can be observed for heterostructures grown on substrates with different crystal orientations. However, only faint deviations of $H_{C}$ can be detected for the pristine $\mathrm{NiFe}$ sample (Figure S2a, Supporting Information). These results suggest that the modulation effect is dominated by the phase change of $\mathrm{VO}_{2}$, rather than by a pure thermal effect of the NiFe layer. The magnetization-temperature $(M-T)$ dependence of the heterostructure sample is then measured as illustrated in Figure 3b. The magnetization increase during the heating process is coordinated with the out-of-plane coercive field shrinking. Interestingly, the $M$ - $T$ curve shows an abnormal valley in the temperature range where the resistance of $\mathrm{VO}_{2}$ drops intensely. This phenomenon can be explained by the phase coexistence of the nonmagnetic layer (for example, the $\mathrm{VO}_{2}$ layer in this work). ${ }^{[29,30]}$ The uniform phase change of $\mathrm{VO}_{2}$ can enhance the magnetization in a gradual way. However, if the phase change is nonuniform, there will be boundaries forming between two different phases of $\mathrm{VO}_{2}$. The $\mathrm{NiFe}$ crystal grains around these boundaries can then be considered as localized magnetic defects and act as pinning centers for magnetic domain wall movements. Thus, the magnetization increase is blocked. As more boundaries form, the magnetization may even decrease until the two types of phases are equal. Consequently, a valley is formed in the $M-T$ curve. The relatively broad temperature range and the flat bottom of this valley also reflect the existence of lattice defects in the $\mathrm{VO}_{2}$ film, which can be confirmed by the TEM results in the later session.

Further experimental evidence indicates that this modulation is an interfacial effect. For heterostructure samples epitaxially deposited on various $\mathrm{TiO}_{2}$ substrates (Figure 3a), the experimental data show that the change in $\mathrm{H}_{C}$ (in-plane) reaches $45 \%$ for $\mathrm{TiO}_{2}$ (100) but only $25 \%$ for $\mathrm{TiO}_{2}(001)$ between the MS and RS. This feature verifies that the modulation depends on the crystalline structure of the $\mathrm{VO}_{2}$ layer. Figure $3 \mathrm{c}$ shows the analysis of the crystal axis conversion of $\mathrm{VO}_{2}$ during its phase change. ${ }^{[21]}$ Remarkably, a more appreciable change can be achieved with the $\mathrm{VO}_{2}$ layer grown on the $\mathrm{TiO}_{2}(100)$ substrate. On the $\mathrm{TiO}_{2}(001)$ substrate, the in-plane lattice constant changes are $\approx 0.4 \%$ and $0.2 \%$ along the $a_{R}$ and $b_{R}$ directions, respectively (Figure $3 d$ ). On the $\mathrm{TiO}_{2}(100)$ substrate, the lattice constant is compressed by $\approx 0.8 \%$ along $c_{R}$, and the change rate between $b_{R}$ and $c_{M}$ reaches $18.5 \%$ with an additional shear force caused by the angle decrease of $\beta$ from $122.6^{\circ}$ to $90^{\circ}$ (Figure 3e; Table S1, Supporting Information). As a result, the interfacial strain effect is enhanced in the $\mathrm{VO}_{2} / \mathrm{TiO}_{2}(100)$ sample. This analysis suggests that the different magnetism modulation mechanisms could be attributed to the different magneto-elastic effect induced by the structure change, in other words, the interfacial strain coupling between $\mathrm{VO}_{2}$ and $\mathrm{NiFe}$. To confirm this conjecture, the modulation 

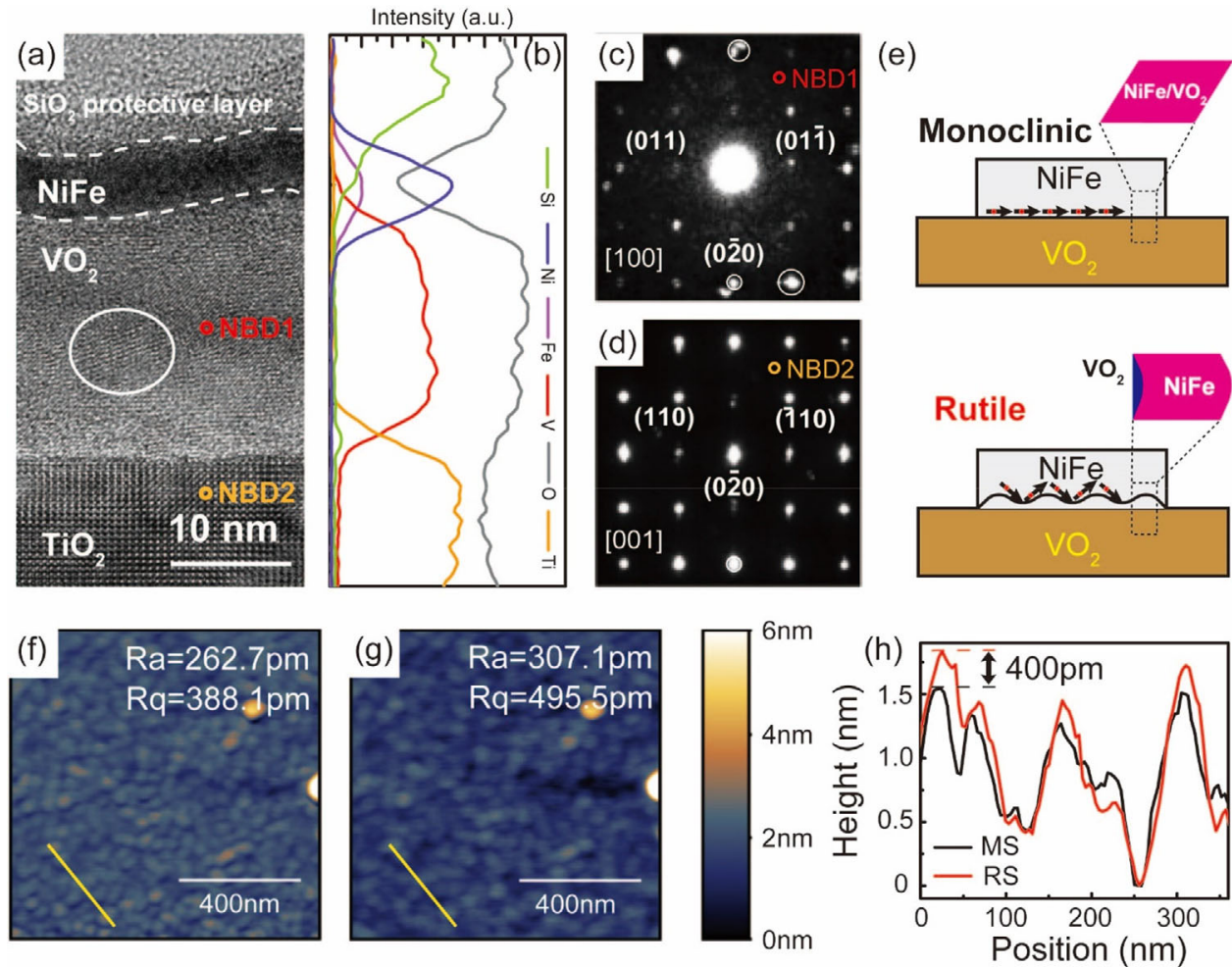

Figure 4. High-resolution transmission electron microscopy (HR-TEM) analysis of the $\mathrm{NiFe} / \mathrm{VO}_{2}$ heterostructure. a) Cross-section HR-TEM image of the $\mathrm{NiFe} / \mathrm{VO}_{2}$ heterostructure deposited on a $\mathrm{TiO}_{2}(100)$ substrate. Some distortion of the crystal orientation can be detected in the $\mathrm{VO}_{2}$ layer, as marked in this figure. b) Energy dispersive spectroscopy (EDS) analysis of different elements (Ti, O, V, Fe, Ni, and $\mathrm{Si}$ ) in the heterostructure. c and d) Nanobeam electron diffraction (NBD) corresponding to NBD1 and NBD2 in (b). e) Schematic diagram of the heterostructure. When the crystal lattice changes, the increased interface roughness causes the spin, which is originally pinned in-plane, to turn to the out-of-plane direction. fand g) Atomic force microscope (AFM) results obtained at 300 and $355 \mathrm{~K}$. The figures also give the average roughness (Ra) and the RMS roughness (Rq) data. A line scanning analysis along the yellow line is given in $(h)$.

effect of heterostructures with different NiFe thicknesses are examined (Figure S2b,c, Supporting Information). The results show that the change in $H_{C}$ reaches $67 \%$ as the thickness decreases to $3 \mathrm{~nm}$ and quickly drops to nearly $0 \%$ as the thickness increases to $10 \mathrm{~nm}$. This result agrees with reports that the magneto-elastic coupling coefficient of $\mathrm{NiFe}$ is anomalously large for thicknesses below $5 \mathrm{~nm} .{ }^{[31]}$ Further engineering of this interfacial strain coupling effect in a $\mathrm{NiFe} / \mathrm{VO}_{2}$ heterostructure could enable optimize modulation performance for various device applications.

Based on the understanding that the magnetism modulation is mainly due to the interfacial strain, we then focus on the origin of the magnetism modulation caused by interfacial strain during the phase change. ${ }^{[32]}$ Recent research has shown that by engineering the surface, perpendicular or other types of magnetic anisotropy can be achieved in ultrathin magnetic films. ${ }^{[33]}$ In our phase change heterostructure, the lattice variation in the specific plane is quite large, which could lead to some periodic bending and spin reorientation of the NiFe film at the interface. ${ }^{[34]} \mathrm{As}^{3}$ suming that it mainly affects the domain wall motion, the mechanism can be understood through a strain-induced anisotropy change.

The coercivity $H_{C}$ is determined by the domain wall energy: ${ }^{[35]}$

$H_{\mathrm{c}} \approx(\partial \gamma / \partial x)_{\max } /\left(8 \pi M_{S}\right)$ where $x$ is the position of the domain wall, $M_{S}$ is the saturation magnetization, and $\gamma$ is the domain wall energy. $\gamma$ can be further written as: ${ }^{[36]}$

$\gamma=4 \sqrt{A\left(K_{1}+\lambda_{\mathrm{s}} \sigma\right)}$

where $A$ is the exchange constant, $K_{1}$ is the crystal anisotropy, $\lambda_{s}$ is the magnetostriction coefficient, and $\sigma$ is the strain applied to the sample. Thus, $H_{C}$ depends on the strain-induced variation in the anisotropy.

As shown in Figure 4a,4b, the cross-section transmission electron microscopy (cross-section TEM) results of the heterostructure on $\mathrm{TiO}_{2}(100)$ substrate verify the existence of thin-film bending in the heterostructure and the absence of obvious intermixing at the interface. Some distortion of the crystal orientation in the $\mathrm{VO}_{2}$ layer can be observed near the bending area (Figure 4a, TEM image; Figure 4c,4d, nanobeam electron diffraction result). It may be caused by the $\mathrm{VO}_{2}$ phase change when cooling from a high growth temperature to room temperature. These structure features may further enhance the interface roughness of $\mathrm{NiFe}$ after the phase change, inducing reorientation of the spin from in-plane to out-of-plane ${ }^{[31]}$ (Figure 4e). The saturation magnetization decrease and coercive force shrinkage are probably caused by 

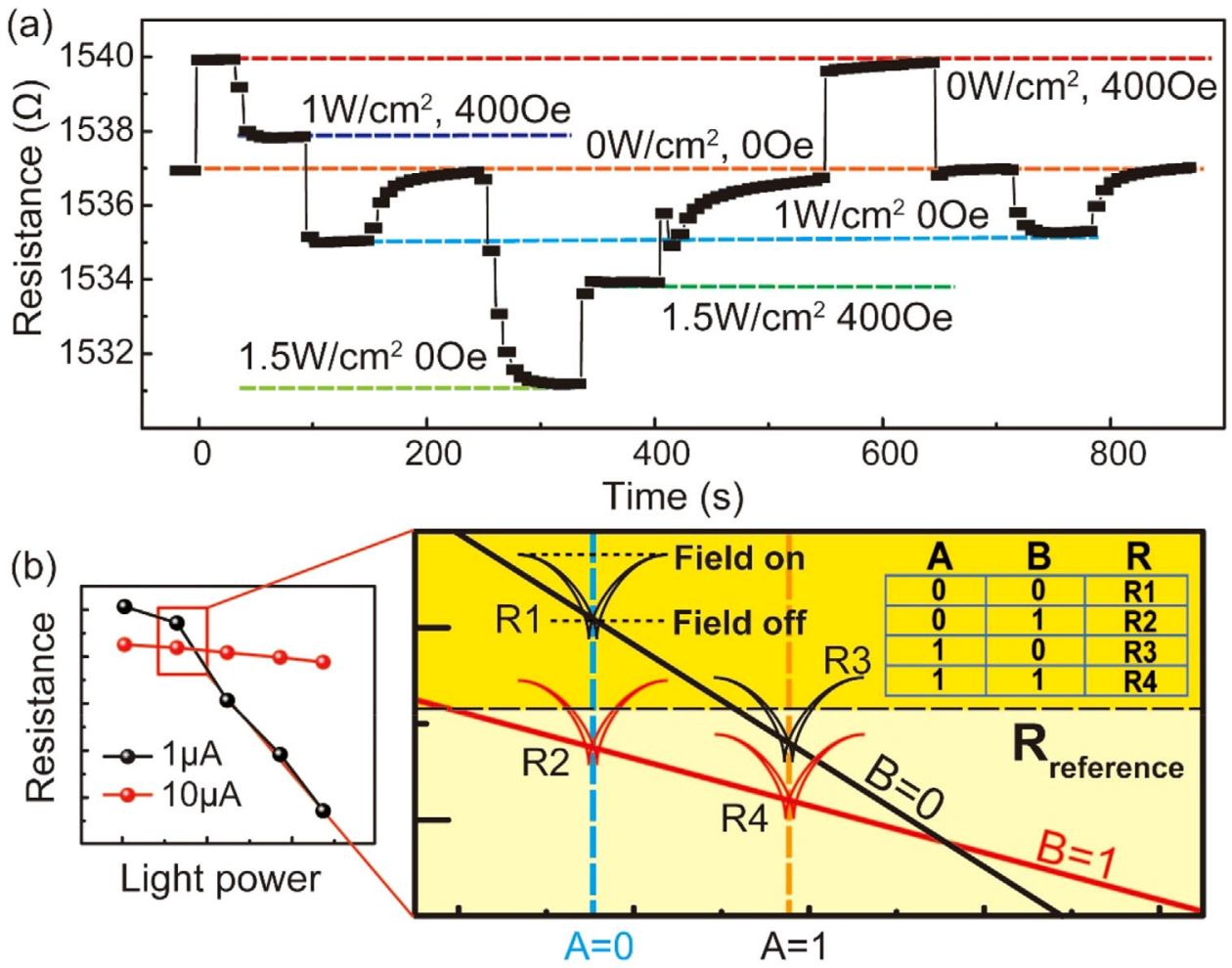

(c)

\begin{tabular}{|c|c|c|}
\hline Field & & NOR \\
\hline$I_{A}$ & $I_{B}$ & $I_{\text {output }}$ \\
\hline 0 & 0 & 1 \\
\hline 0 & 1 & 0 \\
\hline 1 & & 0 \\
\hline 1 & & 0 \\
\hline ield & & NAND \\
\hline$I_{A}$ & $I_{B}$ & $I_{\text {output }}$ \\
\hline 0 & 0 & 1 \\
\hline 0 & 1 & 1 \\
\hline 1 & 0 & 1 \\
\hline 1 & 1 & 0 \\
\hline
\end{tabular}

(d)

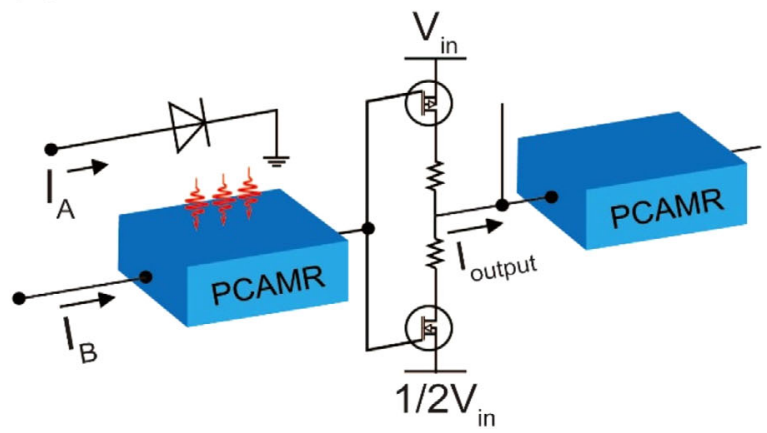

Figure 5. Optically controlled multiresistance states and reconfigurable logics. a) Six different resistance states are realized using different illumination power and magnetic field. b) Two resistance change curves are selected from Figure $2 \mathrm{c}$ to illustrate its dependence on measuring current $(1$ and $10 \mu \mathrm{A})$ when illumination (from 0.5 to $1.6 \mathrm{~W} \mathrm{~cm}^{-3}$ ) applied. The enlarged picture gives the operation settings of the reconfigurable logics. Light illumination (A) and applied current (B) are defined as two kinds of input signals. The corresponding 0 and 1 settings have been indicated by dashed and solid lines, respectively. Four resistance states achieved through the operation of $A$ and $B$ are summarized in the table. The value under same input signals can still be changed by the magnetic field, marked as the field on and field off states by dotted line. This figure also gives the reference resistance $\left(R_{\text {Reference }}\right)$ to determine the output 0 and 1. c) The truth table of NOR and NAND operations. With magnetic field applied and removed, the logic can be reversibly switched between them. d) Circuit schematic to realize device concatenability.

the variation of the distances between individual magnetic grains caused by the bending interface structure.

To further confirm the existence of the phase change induced roughness change, AFM scanning is performed at 300 and $350 \mathrm{~K}$ (Figure 4f,4g). Compared to the sample surface morphology at the MS, an obvious roughness increase can be observed at the RS. Figure $4 \mathrm{~h}$ provides the line scanning result, which shows that the height difference between the two states could reach $400 \mathrm{pm}$. These results are in agreement with the magnetic characteristic results, i.e., the increased $K_{u}$ and enhanced squareness of the outof-plane hysteresis loop.

\section{Multiresistance Realization and Logic Function Implementation}

The magnetic sensitivity (Figure 2a) accompanied with the photosensitivity (Figure 2c) in this heterostructure result in a highly controllable feature of its resistance, which can be adopted for different electronic applications. ${ }^{[37-39]}$ Figure 5a demonstrates an example of six different resistance states achieved via the synergistic control of the light illumination and magnetic field in the PCAMR device. Both the field and illumination enable the reversible, repeatable, and stable modulation of the device 
resistance. The device has been tested over 20 times with different operations continuously, and no resistance shift has been observed in this process, which gives a reference of the device endurance. Further implementation of this phase change effect in other spintronic devices, such as giant magnetoresistance or tunnel magnetoresistance will realize a similar but more appreciable performance.

The unique device resistance dependence on measuring current when illumination applied, can also be used to design reconfigurable logic gates that is highly desired in the information processing. ${ }^{[40,41]}$ Figure $5 \mathrm{~b}$ shows two results of device resistance selected from Figure 2c. As illustrated in the enlarged picture, illumination (defined as A signal) and current (defined as B signal) can be set as two independent inputs to determine the output resistance state. The truth table in Figure $5 \mathrm{~b}$ gives four different states achieved through the operation of $\mathrm{A}$ and B. By setting an appropriate reference resistance $\left(R_{\text {Reference }}\right)$ to determine the output 0 and 1 , a specific logic function is implemented. Notably, the resistance value can still be changed by the magnetic field, marked as the field on and field off states in this figure. So that the logic function can be reversibly switched by magnetic fields, for example, between NOR and NAND as shown in Figure 5c. Such a logic device can be used to perform certain computational tasks, like circuit obfuscation as a hardware encryption to secure information. A circuit schematic is also given in Figure $5 d$, showing a possible strategy to meet the concatenability requirement of logic applications. Apart from the function demos we give above to show the possible applications, it is reasonable to believe that the resistance of the device should be related to the power increasing and decreasing process in the hysteresis range. Such kind of history dependent feature may be used to design beyond von-Neumann computing. ${ }^{43]}$ Reports of beyond micrometer level optical integrations, ${ }^{[42]}$ together with development of electrical spin manipulation methods, such as spin-transfer-torque or spin-orbit-torque effects, may further give birth to a highly integrated phase change spintronic device.

\section{Conclusion}

To conclude, we have combined a strongly correlated electron material and a spintronic material to fabricate new artificial heterostructures in which the spintronic material is endowed with the optical modulation ability of the magnetic and electrical properties by a reversible phase change process. Changes of the electrical conductivity (32\%), coercivity $(37.5 \%)$, and magnetic anisotropy (25\%) have been observed in the $\mathrm{NiFe} / \mathrm{VO}_{2}$ heterostructure. Theoretical analyses and experimental evidence reveal that the magneto-elastic coupling induced by appreciable interfacial strain (up to $18.5 \%$ ) may be the origin of the magnetism modulation. Further device implementations indicate that multiresistance states and reconfigurable logic functions can be achieved in the device. Our work, as a demonstration of optically controlled phase change spintronics, may pave the way for next-generation opto-electronics.

\section{Experimental Section}

Heterostructure and Device Fabrication: The $\mathrm{VO}_{2}$ thin films were grown on (001) and (100) $\mathrm{TiO}_{2}$ substrates using a PLD system ( $\left.\mathrm{KrF}, \lambda=248 \mathrm{~nm}\right)$. The growth of epitaxial $\mathrm{VO}_{2}$ thin films was performed at $500^{\circ} \mathrm{C}$ with $2.0 \mathrm{~Pa}$ of oxygen. The laser fluence and repetition rate were fixed at $4 \mathrm{~J} \mathrm{~cm}^{-2}$ and $2 \mathrm{~Hz}$, respectively. The film thickness was $20 \mathrm{~nm}$ after 7200 deposition pulses. The $\mathrm{Ni}_{80} \mathrm{Fe}_{20}$ films were magnetron-sputtered at room temperature. A total of $5 \mathrm{~nm} \mathrm{SiO}$ was used as a protection layer from oxidation. Raw bilayer samples were patterned by optical lithography (Micro Writer ML Baby, Durham Magneto Optics) followed by argon ion-beam etching into Hall bars with several legs with different spacings. Optical lithography and e-beam evaporation were then used to prepare $\mathrm{Cr}(5 \mathrm{~nm}) / \mathrm{Au}(100 \mathrm{~nm})$ electrodes. Then, $10 \mathrm{~nm}$ of $\mathrm{SiO}_{2}$ was deposited by thermal evaporation on the device as a protection layer.

Characterization and Measurement: The magnetic properties were measured by MOKE (NanoMOKE3, Durham Magneto optics Itd) with a red-light laser (LR-MFJ-660/2000 mW, Changchun Laser Technology Co.) heat source and SQUID (MPMS3, Quantum Design, Inc.) at room and high temperatures. The PCAMR devices were measured by normal fourterminal methods with Keithley 6221 and Keithley 2182 sourcing and measuring units, respectively. Meanwhile, an in-plane electromagnet (East Changing Co. China) provided magnetic fields with proper directions.

\section{Supporting Information}

Supporting Information is available from the Wiley Online Library or from the author.

\section{Acknowledgements}

G.W. and X.L. contributed equally to this work. The authors thank Huaiwen Yang, Zhaohao Wang, Jiwei Hou, Yuan Cao, and Runrun Hao for their help in device fabrication. This work was supported by the National Natural Science Foundation of China (nos. $51602013,11804016,61704005$, and 61571023), Young Elite Scientists Sponsorship Program by China Association for Science and Technology (CAST) (no. 2018QNRC001), the International Collaboration 111 Project (no. B 16001), the China Postdoctoral Science Foundation (no. 2018M631296), the Fundamental Research Funds for the Central Universities of China, and the Beijing Advanced Innovation Center for Big Data and Brain Computing (BDBC).

\section{Conflict of Interest}

The authors declare no conflict of interest.

\section{Keywords}

magnetoresistance, optical control, phase change, spintronics, $\mathrm{VO}_{2}$

Received: August 27, 2019

Revised: November 22, 2019

Published online: January 9, 2020

[1] S. A. Wolf, Science 2001, 294, 1488.

[2] C. Chappert, A. Fert, F. N. Van Dau, Nat. Mater. 2007, 6, 813.

[3] X. Lin, W. Yang, K. L. Wang, W. Zhao, Nat. Electron. 2019, 2, 274.

[4] P. V. Ong, N. Kioussis, P. K. Amiri, K. L. Wang, Sci. Rep. 2016, 6, 29815. 
[5] T. Maruyama, Y. Shiota, T. Nozaki, K. Ohta, N. Toda, M. Mizuguchi, A A. Tulapurkar, T. Shinjo, M. Shiraishi, S. Mizukami, Y. Ando, Y. Suzuki, Nat. Nanotechnol. 2009, 4, 158.

[6] D. H. Auston, Appl. Phys. Lett. 1975, 26, 101.

[7] S. Mangin, M. Gottwald, C.-H. Lambert, D. Steil, V. Uhlír, L. Pang, M. Hehn, S. Alebrand, M. Cinchetti, G. Malinowski, Y. Fainman, M. Aeschlimann, E. E. Fullerton, Nat. Mater. 2014, 13, 286.

[8] R. Ramesh, N. A. Spaldin, Nat. Mater. 2007, 6, 21.

[9] S. M. Wu, S. A. Cybart, P. Yu, M. D. Rossell, J. X. Zhang, R. Ramesh, R. C. Dynes, Nat. Mater. 2010, 9, 756.

[10] G. Wei, L. Wei, D. Wang, Y. Chen, Y. Tian, S. Yan, L. Mei, J. Jiao, Appl. Phys. Lett. 2017, 110, 062404.

[11] N. Lu, P. Zhang, Q. Zhang, R. Qiao, Q. He, H.-B. Li, Y. Wang, J. Guo, D. Zhang, Z. Duan, Z. Li, M. Wang, S. Yang, M. Yan, E. Arenholz, S. Zhou, W. Yang, L. Gu, C.-W. Nan, J. Wu, Y. Tokura, P. Yu, Nature 2017, 546, 124.

[12] U. Bauer, L. Yao, A. J. Tan, P. Agrawal, S. Emori, H. L. Tuller, S. Van Dijken, G. S. D. Beach, Nat. Mater. 2015, 14, 174.

[13] P. Liu, X. Lin, Y. Xu, B. Zhang, Z. Si, K. Cao, J. Wei, W. Zhao, Materials 2017, 11, 47.

[14] T. L. Koch, U. Koren, IEEE J. Quantum Electron. 1991, 27, 641.

[15] X. Sun, S. Vélez, A. Atxabal, A. Bedoya-Pinto, S. Parui, X. Zhu, R. Llopis, F. Casanova, L. E. Hueso, Science 2017, 357, 677.

[16] A. V Kimel, M. Li, Nat. Rev. Mater. 2019, 4, 189.

[17] M. Imada, A. Fujimori, Y. Tokura, Rev. Mod. Phys. 1998, 70, 1039.

[18] M. Nakano, K. Shibuya, D. Okuyama, T. Hatano, S. Ono, M. Kawasaki, Y. Iwasa, Y. Tokura, Nature 2012, 487, 459

[19] S. Lee, K. Hippalgaonkar, F. Yang, J. Hong, C. Ko, J. Suh, K. Liu, K. Wang, J. J. Urban, X. Zhang, C. Dames, S. A. Hartnoll, O. Delaire, J. Wu, Science 2017, 355, 371.

[20] J. Jeong, N. Aetukuri, T. Graf, T. D. Schladt, M. G. Samant, S. S. P. Parkin, Science 2013, 339, 1402.

[21] Z. Tao, T.-R. T. Han, S. D. Mahanti, P. M. Duxbury, F. Yuan, C.-Y. Ruan, K. Wang, J. Wu, Phys. Rev. Lett. 2012, 109, 166406.

[22] A. Cavalleri, T. Dekorsy, H. H. W. Chong, J. C. Kieffer, R. W. Schoenlein, Phys. Rev. B 2004, 70, 161102.

[23] C. Kübler, H. Ehrke, R. Huber, R. Lopez, A. Halabica, R. F. Haglund, A. Leitenstorfer, Phys. Rev. Lett. 2007, 99, 116401.
[24] K. Liu, S. Lee, S. Yang, O. Delaire, J. Wu, Mater. Today 2018, 21, 875.

[25] J. G. Ramirez, T. Saerbeck, S. Wang, J. Trastoy, M. Malnou, J. Lesueur, J. P. Crocombette, J. E. Villegas, I. K. Schuller, Phys. Rev. B 2015, 91, 205123.

[26] S. Ikeda, K. Miura, H. Yamamoto, K. Mizunuma, H. D. Gan, M. Endo, S. Kanai, J. Hayakawa, F. Matsukura, H. Ohno, Nat. Mater. 2010, 9, 721.

[27] B. N. Engel, C. D. England, R. A. Van Leeuwen, M. H. Wiedmann, C. M. Falco, Phys. Rev. Lett. 1991, 67, 1910.

[28] G. Wei, X. Lin, Z. Si, N. Lei, Y. Chen, S. Eimer, W. Zhao, Appl. Phys. Lett. 2019, 114, 012407.

[29] J. Lauzier, L. Sutton, J. de la Venta, J. Appl. Phys. 2017, 122, 173902.

[30] J. de la Venta, S. Wang, T. Saerbeck, J. G. Ramírez, I. Valmianski, I. K. Schuller, Appl. Phys. Lett. 2014, 104, 062410.

[31] O. Song, C. A. Ballentine, R. C. O'Handley, Appl. Phys. Lett. 1994, 64, 2593.

[32] J. P. Reekstin, J. Appl. Phys. 1967, 38, 1449.

[33] O. A. Tretiakov, M. Morini, S. Vasylkevych, V. Slastikov, Phys. Rev. Lett. 2016, 119, 077203.

[34] P. Graczyk, R. Schäfer, B. Mroz, J. Phys. D: Appl. Phys. 2015, 48, 425002.

[35] I. J. Garshelis, J. Appl. Phys. 1993, 73, 5629

[36] C. Garrett, P. Holland, W. J. Geerts, D. Ragan, A. Dubey, S. Rios, A. K. Bandyopadhyay, J. Appl. Phys. 2003, 93, 8624.

[37] H. Ma, J. Hou, X. Wang, J. Zhang, Z. Yuan, L. Xiao, Y. Wei, S. Fan, K. Jiang, K. Liu, Nano Lett. 2017, 17, 421.

[38] L. Xiao, H. Ma, J. Liu, W. Zhao, Y. Jia, Q. Zhao, K. Liu, Y. Wu, Y. Wei, S. Fan, K. Jiang, Nano Lett. 2015, 15, 8365

[39] K. Dong, S. Hong, Y. Deng, H. Ma, J. Li, X. Wang, J. Yeo, L. Wang, S. Lou, K. B. Tom, K. Liu, Z. You, Y. Wei, C. P. Grigoropoulos, J. Yao, J. Wu, Adv. Mater. 2018, 30, 1703878

[40] X. Lin, L. Su, Z. Si, Y. Zhang, A. Bournel, Y. Zhang, J. Klein, A. Fert, W. Zhao, Phys. Rev. Appl. 2017, 8, 034006.

[41] C. Wan, X. Zhang, Z. Yuan, C. Fang, W. Kong, Q. Zhang, H. Wu, U. Khan, X. Han, Adv. Electron. Mater. 2017, 3, 1600282.

[42] C. S. Davies, J. Janušonis, A. V Kimel, A. Kirilyuk, A. Tsukamoto, T. Rasing, R. I. Tobey, J. Appl. Phys. 2018, 123, 213904.

[43] C. D. Wright, P. Hosseini, J. A. V. Diosdado, Adv. Funct. Mater. 2013, 23,2248 